\title{
Time to switch to ferroelectronics?
}

\author{
Ferroelectric field-effect transistors could play a key role in the development of data-centric computing hardware.
}

ast month, we highlighted the challenges facing research into negative capacitance field-effect transistors and their use in advanced semiconductor technology nodes ${ }^{1}$. Questions remain over the future of these devices, but at their core are ferroelectric materials, and the switching properties of such materials are valuable in a range of engineering applications. Ferroelectrics have an intrinsic lattice polarization that can be switched by an external electric field, behaviour that appears as a hysteresis loop in the relationship between the applied electric field and the stored charge. Unlike the polarization observed in typical dielectrics, ferroelectrics remain polarized after removal of the electric field and thus exhibit a non-volatile-like behaviour.

The first ferroelectrics studied were fragile and of limited practical value, but in the 1940s the discovery of barium titanate - a more robust material with properties suited to engineering applications - led to the emergence of the electronic ceramics industry ${ }^{2}$. These ferroelectrics could though only be manufactured in bulk, and had high coercive fields (typically many $\mathrm{kV} \mathrm{cm}^{-1}$ ). This meant that early applications focused on the use of capacitors and transducers, which take advantage of the high dielectric permittivity or piezoelectric properties (accumulation of electric charge in response to applied mechanical stress) of the ferroelectrics.

By the 1970s, thin-film deposition techniques had been developed that allowed sub-micrometre-thick layers to be formed and used in electronic devices. With such thin films, switching could be achieved using voltages compatible with the broader integrated circuit industry, which by this time was expanding rapidly. Early laboratory demonstrations used barium titanate as the gate dielectric in a metal-oxide-semiconductor field-effect transistor structure in order to create a ferroelectric field-effect transistor ${ }^{3}$; the switching behaviour of the ferroelectric led to a hysteresis in the transfer characteristics of the transistor, thus acting as a non-volatile memory.

By the late 1970s, it was possible to monolithically integrate ferroelectric capacitors and silicon transistors ${ }^{4}$. Later, the fabrication of memory circuits that used a similar layout to volatile dynamic

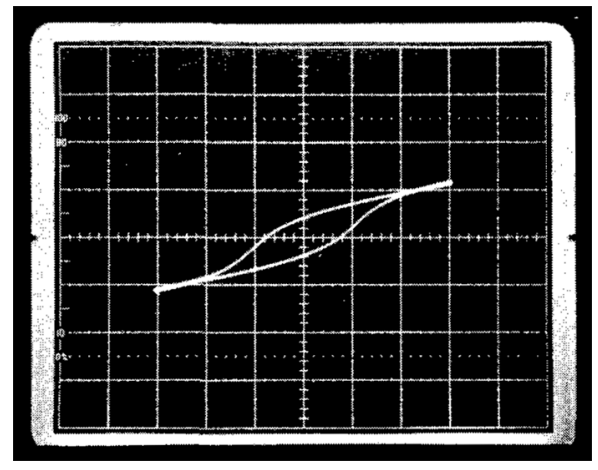

Ferroelectric hysteresis loop of a barium titanate film, taken from work on ferroelectric field-effect memory devices in the $1970 \mathrm{~s}^{3}$. Image reproduced with permission from ref. ${ }^{3}$, American Institute of Physics.

random-access memory - where the drain electrode of a transistor connects to a ferroelectric capacitor - became a common approach ${ }^{5}$. And this circuit design saw commercial success with companies such as Ramtron (acquired by Cypress Semiconductor in 2012) and Texas Instruments. However, ferroelectric field-effect transistors were not widely adopted as the deposition processes for the ferroelectric oxides were incompatible with smaller silicon technology nodes.

Around 2007 the situation changed. Transistor scaling had led to the use of increasingly thin silicon oxide dielectrics, which were needed to maintain device performance but led to increased leakage current that affected efficiency and reliability. And thus, Intel announced a change to hafnium oxide ${ }^{6}:$ a gate material with a high dielectric constant that could be deposited at a greater thickness to limit leakage, but still maintain electrostatic coupling and device performance. The use of hafnium oxide dielectrics in advanced silicon nodes is now well established, and in 2011 it was discovered that with suitable doping hafnium oxide also exhibits ferroelectric behaviour ${ }^{7}$.

In a Perspective article in this issue of Nature Electronics, Asif Khan, Ali Keshavarzi and Suman Datta discuss how hafnium oxide has made ferroelectric field-effect transistors a viable technology for the advanced microelectronics industry offering a new approach to electronic devices they term ferroelectronics. The energy efficiency, speed and cell sizes of the devices make them suitable for use in a variety of specialized memory circuit applications, and by integrating logic and memory in a single device they could enable architectures that overcome the 'memory wall' of conventional computing systems: that is, the inefficient shuffling of data between memory and processing units.

Khan and colleagues - who are based at Georgia Institute of Technology, Stanford University, and the University of Notre Dame - also highlight that there are opportunities beyond binary memory applications. Ferroelectric field-effect transistors can be engineered to display plasticity and multiple logic levels, behaviours that are desirable for use in artificial neural networks and neuromorphic computing. Currently, most artificial neural networks are run as simulations on conventional computing systems. The development of more efficient neuromorphic hardware - that physically mimics the behaviour of biological systems will be needed to meet the future demands of machine learning and artificial intelligence.

Many of the biggest challenges facing electronics and computing - with its increasing focus on data-centric applications - are at the architecture level. Although there is still work to be done at the technology level, particularly from a neuromorphic device perspective, hafnium oxide is now a proven technology and ferroelectric field-effect transistors with memory functionality have been demonstrated at advanced technology nodes ${ }^{8}$. And thus, if ferroelectronics is to play a key role in the future of hardware, the use of ferroelectric field-effect transistor technology in very-large-scale-integration applications is an important next step.

Published online: 19 October 2020 https://doi.org/10.1038/s41928-020-00496-3

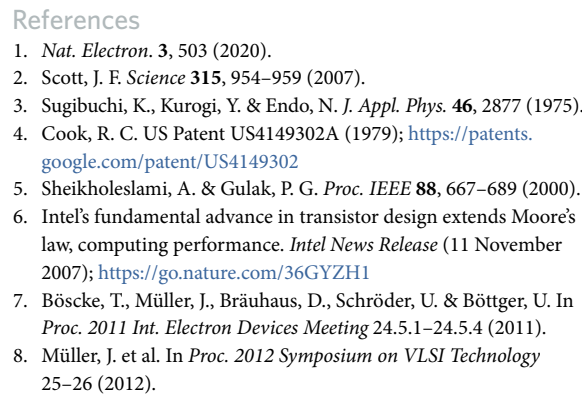

